\section{Time-resolved IR-absorption spectroscopy of hot-electron dynamics in satellite and upper conduction bands in GaP}

\author{
M. A. Cavicchia and R. R. Alfano \\ Institute for Ultrafast Spectroscopy and Lasers, New York State Center for Advanced Technology \\ for Ultrafast Photonic Materials and Applications, Physics Department, The City College and \\ The Graduate School of the City University of New York, New York, New York 10031 \\ (Received 3 November 1994)
}

\section{$A / x$ \\ $1 N-25-C R$ \\ $(C, w)$ \\ $\therefore 30$}

\begin{abstract}
The relaxation dynamics of hot electrons in the $X_{6}$ and $X_{7}$ satellite and upper conduction bands in GaP was directly measured by femtosecond UV-pump-IR-probe absorption spectroscopy. From a fit to the induced IR-absorption spectra the dominant scattering mechanism giving rise to the absorption at early delay times was determined to be intervalley scattering of electrons out of the $X_{7}$ upper conduction-band valley. For long delay times the dominant scattering mechanism is electron-hole scattering. Electron transport dynamics of the upper conduction band of $\mathrm{GaP}$ has been time resolved.
\end{abstract}

The relaxation of hot-electron distributions has been very well characterized in various semiconductors by ultrafast spectroscopy. Relatively little work has been performed in $\mathrm{GaP}$, since $\mathrm{GaAs}$ and $\mathrm{Si}$ have been the systems of choice for study due to their technological importance in optical and electronic applications. GaP has previously been considered as a material for fast mid-IR photodetection. ${ }^{1}$ GaP transport properties are now becoming important for its use in complex alloy structures, e.g., $\mathrm{In}_{1-x} \mathrm{Ga}_{x} \mathrm{As}_{y} \mathrm{P}_{1-y} / \mathrm{InP}$ heterostructure lasers ${ }^{2}$ and $\mathrm{In}_{1-x} \mathrm{Ga}_{x} \mathrm{P} / \mathrm{In} \mathrm{P}$ solar cells. ${ }^{3}$ Intervalley scattering is expected to be one of the dominant scattering mechanisms for hot, high-density electrons in bulk and quantum-well semiconductors, limiting their role in devices. 4 In addition, the upper conduction band may play an important role in the transport properties of hot, high-density electrons in these devices and other nanostructures under high-field conditions.

In this article, we present an experimental study of the intervalley relaxation of hot electrons in the satellite and upper conduction-band $X_{6}$ and $X_{7}$ valleys in GaP using time-resolved UV-pump-IR-probe absorption spectroscopy. From the induced absorption spectra the dominant scattering mechanisms, the transient mobility, and the deformation potential for intervalley scattering between the $X_{6}$ and $X_{7}$ valleys were determined for the hot electrons in the satellite and upper conduction-band valleys.

In the experiment, 500-fs pump pulses were obtained from the fundamental wavelength $(585 \mathrm{~nm})$ or the second harmonic $(293 \mathrm{~nm})$ of a synchronously pumped, modelocked Rhodamine $6 \mathrm{G}$ dye laser. The 500 -fs, tunable IR probe pulses were generated by difference frequency mixing of the fundamental pulse with a supercontinuum pulse generated in methanol, and were used to measure the induced IR absorption. The pump and probe pulses were loosely focused onto a $350-\mu \mathrm{m}$ aperture to obtain uniform intensities across the sample. A fraction of the probe pulse was measured before passing through the sample to be used as a reference to minimize shot-to-shot intensity fluctuations. The temporal resolution of this system is $700 \mathrm{fs}^{6}$ The sample used was a 0.5 -mm-thick, n-type GaP crystal doped with Se at a concentration of $\sim 10^{17} \mathrm{~cm}^{-3}$. The measurements were done at room temperature.

Measurements were made of the transient IR absorption at wavelengths between 2.5 and $5.5 \mu \mathrm{m}$, when the sample was photoexcited with either $\lambda_{\text {pump }}=293 \mathrm{~nm}$ or $\lambda_{\text {pump }}=585 \mathrm{~nm}$. The temporal behavior of the electron population in the $X_{6}$ and $X_{7}$, second conduction-band valleys was determined by monitoring the transient IRabsorption changes due to the photoexcited free carrier and due to $X_{6} \rightarrow X_{7}$ interconduction band absorption.

The intense UV (293 nm) pump pulse was used to photoexcite electrons from the heavy-hole light-hole, and split-off-hole valence bands to the $\Gamma_{6}$, central valley of the first conduction band by direct absorption. These photoexcited electrons obtained an average kinetic energy of $\sim 0.8 \mathrm{eV}$, sufficient for intervalley scattering from the $\Gamma_{6}$ valley to both the $X_{6}$, first conduction-band satellite valley, and the $X_{7}$, upper conduction-band valley as well as the $L_{6}$ first conduction-band valley. Once electrons scatter to the satellite valleys they may scatter back and forth to the $\Gamma_{6}$ valley, scatter between the satellite valleys, or relax within a given valley by emitting LO phonons. The effect of these relaxation processes and electron thermalization is to put a large $\left(\sim 10^{20} \mathrm{~cm}^{-3}\right)$ thermalized population of hot electrons in the $X$ valleys within the risetime of the induced IR absorption ( $\leqslant 2 \mathrm{ps}$ ). Subsequent energy relaxation occurs, and the electrons cool down and are redistributed among the conductionband valleys by intervalley scattering (see the inset of Fig. 1). The weak, time-delayed IR probe pulse was used to monitor the induced absorption as a function of pumpprobe delay time.

The induced IR-absorption spectra measured under UV photoexcitation at pump-probe delay times of 2 and $30 \mathrm{ps}$ are displayed in Fig. 1. The solid lines in Fig. 1 are a least-squares fit of the spectra using a model that includes contributions to the induced IR absorption from free-carrier absorption (FCA) by the photoexcited elec- 


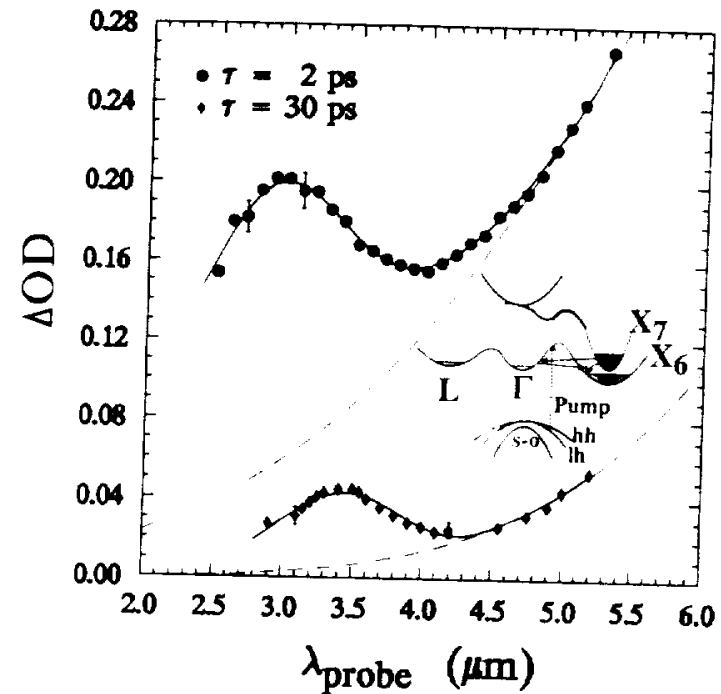

FIG. 1. Induced IR-absorption spectra in GaP, $\lambda_{\text {pump }}=293$ $\mathrm{nm}$, at delay times of $\tau=2$ and $30 \mathrm{ps}$. The solid lines are lineshape fits of the data. The dashed lines are the calculated FCA spectra. The inset is a schematic diagram of the band structure of $\mathrm{GaP}$ showing the pump and probe transitions and relevant intervalley scattering transitions.

trons and holes, ${ }^{7}$ direct interconduction-band absorption (DIBA) by electrons in the $X_{6}$ valley to the $X_{7}$ valley, ${ }^{8}$ and indirect interconduction-band absorption (IIBA) by electrons in the $X_{6}$ valley to the $\Gamma_{6}$ or $L_{6}$ valleys.? Intervalence-band absorption has been shown to be negligible at the probe wavelengths used in this study. 9 The free-carrier contribution, which dominates at longer wavelengths, rises as $\triangle O D_{\mathrm{FCA}}(\lambda) \propto \lambda^{P}$ (Ref. 10). The best fit to the spectra at $\tau=2 \mathrm{ps}$ is given by $p=2.5$, but for later times, at $\tau=30$ ps the best fit is given by $p=3.5$. This effect will be discussed below.

The direct $X_{6} \rightarrow X_{7}$ absorption spectrum of this allowed, momentum-conserving transition is calculated using perturbation theory. The absorption change can be written, after integration over the electron wave vector,
as $^{8}$

$$
\begin{aligned}
\Delta O D_{\mathrm{DIBA}}(\lambda) \propto & \left(\frac{h c}{\lambda}-\Delta E_{X_{6}}^{\prime}, X,\right)^{1 / 2} m_{r}^{2 / 3} \\
& \times\left[f_{6}\left(E_{6}, E_{f 6}, T_{e}\right)-f_{y}\left(E_{7}, E_{f 7}, T_{e}\right)\right],
\end{aligned}
$$

where $f_{6}$ and $f_{7}$ are the Fermi-Dirac distributions for electrons in the $X_{6}$ and $X_{7}$ valleys, respectively, $E_{f 6}$ and $E_{f 7}$ are the quasi-Fermi energies for the populations in the $X_{6}$ and $X_{7}$ valleys, respectively, $\Delta E_{X_{6}, X_{7}}^{\prime}$ is the renormalized energy gap between the $X_{6}$ and $X_{7}$ valley mini$\mathrm{ma}^{11} T_{e}$ is the electron temperature, and $m_{r}$ is the reduced effective mass $m_{r}=m_{6} m_{7} /\left(m_{6}-m_{7}\right)$, where $m_{6}$ and $m_{7}$ are the density of states effective masses of the $X_{6}$ and $X_{7}$ are the density of states effective masses of the $X_{6}$ and $X_{7}$ valleys, respectively. For GaP, $m_{6}=1.33 m_{e}$ and $m_{7}=0.72 m_{e}$, ${ }^{12}$ where $m_{e}$ is the free-electron mass. The energies of the optically coupled states, $E_{6}$ and $E_{7}$, at the probe wavelength $\lambda$, can be expressed in terms of the effective masses and $\Delta E_{X_{6}, X_{7}}^{\prime}$ as

$$
E_{6,7}(\lambda)=\left(m_{r} / m_{6,7}\right)\left(h c / \lambda-\Delta E_{X_{6}, X_{7}}^{\prime}\right) \text {. }
$$

From our fit to the induced spectrum we can extract the hot-electron temperature $T_{e}$ and the electron energy distributions in the $X_{6}$ and $X_{7}$ valleys, $n_{6}\left(E_{6}, E_{f 6}, T_{e}\right)$ and $n_{7}\left(E_{6}, E_{f 6}, T_{e}\right)$, respectively. ${ }^{13}$ It was found that at 2 ps essentially all of the electrons have been transferred to the $X_{6}$ and $X_{7}$ valleys from the $\Gamma_{6}$ valley. The DIBA is characterized by a large Burstein-Moss shift ${ }^{10}$ due to the degeneracy of the electron population in the upper conduction-band, $X_{7}$, valley. Between 2 and 30 ps, the Burstein-Moss shift of the DIBA is significantly reduced, indicating that the electron population in the $X_{7}$ valley has been reduced.

The theoretical fits to the induced IR-absorption spectra show that probe photons with a wavelength of $4.7 \mu \mathrm{m}$ are not energetic enough to cause electron interband transitions, i.e., only FCA is present. Figure 2 shows the completely time-resolved change of IR absorption when $\lambda_{\text {pump }}=293 \mathrm{~nm}$ and $\lambda_{\text {probe }}=4.7 \mu \mathrm{m}$. The temporal behavior is characterized by a rapid rise and peak in the absorption, followed by a fast decay component ( $\sim 5 \mathrm{ps})$, and finally a slow decay component that is flat during the experimental time range of 100 ps.

To eliminate transfer to the upper conduction-band, $X_{7}$ valley, a visible $(585 \mathrm{~nm})$ pump pulse was used with $\lambda_{\text {probe }}=4.7 \mu \mathrm{m}$. The peak at early delay times, detected with $293-\mathrm{nm}$ excitation, was not observed with $585-\mathrm{nm}$ excitation. This result is shown in the inset of Fig. 2, and is characterized by a rapid rise in absorption. Only a slow decay component that is almost flat during the experimental time range of $100 \mathrm{ps}$ is observed. With $\lambda_{\text {pump }}=585 \mathrm{~nm}$, electrons are photoexcited only to the $X_{6}$ valley by an indirect absorption, and do not obtain

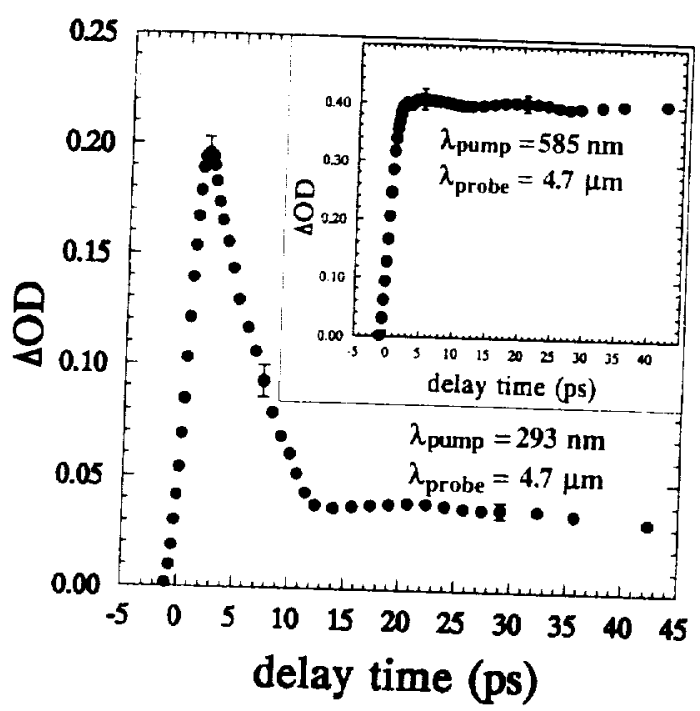

FIG. 2. Transient induced IR-absorption when $\lambda_{\text {pump }}=293$ $\mathrm{nm}$ and $\lambda_{\text {probe }}=4.7 \mu \mathrm{m}$. The excess absorption is due to electrons in the $X$, valley. The inset is the transient IR absorption when $\lambda_{\text {pump }}=585 \mathrm{~nm}$ and $\lambda_{\text {mine }}=4.7 \mu \mathrm{m}$. 
sufficient kinetic energy to undergo intervalley scattering to the $X_{7}$ valley. Therefore, no redistribution occurs between the $X_{6}$ and $X_{7}$ valleys.

In the classical Drude model, FCA at a particular wavelength is related to the carrier density $N$, the carrier effective mass $m^{*}$, and the carrier mobility $\mu$, by $\alpha_{\mathrm{FCA}} \propto N /\left(m^{* 2} \mu\right)$. The mobility is given by $\mu=e\langle\tau\rangle / m^{*}$, where $e$ is the charge of the carrier and $\langle\tau\rangle$ is the average carrier momentum relaxation time. ${ }^{14}$ Therefore, electrons in different valleys, with different effective masses and different mobilities, will have different free-carrier absorption cross sections. This effect has been seen experimentally as a time-dependent free-carrier absorption due to the intervalley relaxation of electrons among the different conduction-band valleys in GaAs. ${ }^{15,16}$ It is expected that the mobility of the electrons residing in the upper conduction-band, $X_{7}$, valley will be significantly reduced due to strong, relatively unscreened, intervalley scattering to the higher effectivemass $X_{6}$ valley. ${ }^{16}$ The hole contribution to FCA is expected to be negligible due to degeneracy and screening. ${ }^{16}$ On the basis of all of the above considerations, the excess FCA at early delay times is attributed to the transient electron population in the upper conduction-band, $X_{7}$ valley.

The temporal behavior of the electron population in the upper conduction-band, $X_{7}$, valley, $N_{7}(t)$, is obtained directly from the excess FCA at early delay times ${ }^{16}$ by deconvoluting transient FCA due to the $X_{7}$ electrons with the probe-pulse temporal profile. This result is shown in Fig. 3. These growth and decay times of the transient electron population in the $X_{7}$ valley in GaP have been time resolved. The $X_{7}$ valley electron population peaks at $2.0 \mathrm{ps}$, and has a decay time of $4.5 \mathrm{ps}$.

In the Drude model, the induced FCA is inversely proportional to the mobility and effective mass of the excited carriers. This classical theory, which predicts that $\alpha_{\text {FCA }} \propto \lambda^{2}$, has been shown to fit FCA for wavelengths $\gtrsim 10 \mu \mathrm{m}$ accurately. ${ }^{15}$ For our range of probe wave-

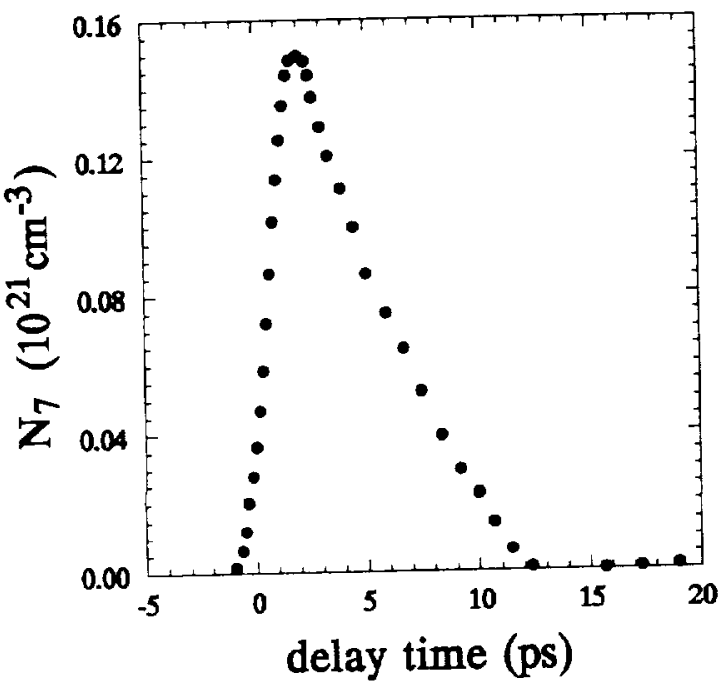

FIG. 3. Transient electron population in the $X_{7}$ valley. $N_{7}(t)$ (circles) is obtained as explained in the text. lengths it is necessary to perform a full quantummechanical calculation including all of the possible scattering mechanisms which contribute to the mobility, and then average over the carrier energy distributions. ${ }^{14}$ The results of this calculation will be compared with the experimental FCA magnitude and spectral dependence to obtain the transient mobility of the carriers and the dominant scattering mechanisms which contribute to the mobility.

FCA is a second-order process where a free carrier in state $k$ first makes a transition to a virtual state $k^{\prime}$ by absorption of a photon, and then makes a transition to the final state $k$ " by absorption or emission of a phonon which conserves crystal momentum, or by the reverse process. Using second-order perturbation theory the absorption coefficient may be written as

$\alpha_{\mathrm{FCA} \pm}=\frac{4 \hbar \mu_{0} c V}{(2 \pi)^{6} n A_{0}^{2} \omega} \int d^{3} k d^{3} k^{\prime \prime} S_{ \pm} f(k)\left[1-f\left(k^{\prime \prime}\right)\right]$

where $n$ is the index of refraction; $A_{0}$ is the amplitude of the vector potential of the probe pulse; $\mu_{0}$ is the permeability in vacuum; and $\omega$ is the probe photon frequency, where $f(k)$ and $f\left(k^{\prime \prime}\right)$ are the occupation probabilities of the initial and final free carrier states, respectively. The transition probability $S_{ \pm}$is given by

$S_{ \pm}=\frac{2 \pi}{\hbar}\left[\frac{\left|H_{k^{\prime} k}\right|^{2}\left|H_{k^{\prime \prime} k^{\prime}}\right|_{ \pm}^{2}}{\hbar^{2} \omega^{2}}\right] \delta\left(E^{\prime}-E-\hbar \omega \pm \hbar \omega_{q}\right)$,

where $H_{k^{\prime \prime} k^{\prime}}$ is the matrix element of the Hamiltonian for phonon emission $(+)$ or absorption $(-), H_{k^{\prime} k}$ is the matrix element for photon absorption, $\omega_{q}$ is the phonon frequency with wave vector $q$, and $E$ and $E^{\prime}$ are the initial and final carrier energies, respectively.

Equations (3) and (4) and the appropriate scattering matrix elements are used to calculate the wavelength dependence for the possible types of carrier scattering. This model takes into account the many-valley and multiple-conduction-band nature of the band structure. The scattering mechanisms included were polar-opticalphonon scattering, intervalley deformation-potential scattering by zone-edge LO and LA phonons, intravalley deformation-potential scattering by long-wavelength LO, LA, and TA phonons, and electron-hole scattering, which may have an influence on the mobility. ${ }^{15}$ The matrix element for electron-hole scattering was obtained from the matrix element for ionized-impurity scattering, with the electron mass replaced by the electron-hole reduced mass. ${ }^{17}$

The results of this calculation show that the $X_{6}$ and $X_{7}$ valley electrons make the main contributions to the FCA at 2 ps. At 30 ps most of the electrons have relaxed to the lower-energy $X_{6}$ valley, and make the main contribution to FCA for long delay times. At 2 ps the absorption at $\lambda_{\text {probe }}=4.7 \mu \mathrm{m}$ due to the $X_{7}$ valley electrons is about 4.1 times that of the absorption due to the $X_{6}$ valley electrons. This value agrees well with the ratio of the magnitude of the excess absorption at 2 ps relative to the magnitude of the absorption at $30 \mathrm{ps}$ for $\lambda_{\text {probe }}=4.7 \mu \mathrm{m}$. The 
hole contribution to the FCA is negligible due to degeneracy and screening. The dominant scattering mechanisms at 2 ps are intervalley scattering of $X_{7}$ valley electrons into the $X_{6}$ valley, which dominates the relaxation of the $X_{7}$ valley electrons, and electron-hole scattering. The combination of these two types of scattering mechanisms give rise to a $\lambda^{2.5}$ dependence of the FCA spectrum. It may be noted that polar-optical-phonon scattering also gives rise to the same wavelength dependence, but the magnitude of the absorption is much too small due to strong screening of this scattering process by the high-density electron-hole plasma. ${ }^{18}$ On the other hand, intervalley deformation-potential scattering is relatively unscreened. At $30 \mathrm{ps}$ the dominant scattering mechanism is electron-hole scattering. Electron-hole scattering gives rise to a $\lambda^{3.5}$ dependence of the FCA spectrum, in agreement with the spectrum measured at $30 \mathrm{ps}$.

The dominant scattering mechanism for the $X_{7}$ valley electron population, at $2 \mathrm{ps}$, was found to be intervalley scattering to the $X_{6}$ valley by LO and LA phonons. The transient mobility of the $X_{7}$ population can be estimated from the calculated intervalley scattering rate. The matrix element for the intervalley scattering is ${ }^{14}$

$$
\left|H_{k^{\prime \prime} k^{\prime}}\right|_{ \pm}=D_{X_{6} X_{7}}\left[\left(N_{q}+\frac{1}{2} \mp \frac{1}{2}\right) \hbar / 2 \rho V \omega_{q}\right]^{1 / 2},
$$

where $N_{q}$ is the Bose-Einstein occupation number for the relevant phonons with wave vector $q, \rho$ is the mass density, $V$ is the crystal volume, and $D_{X_{6} X_{7}}$ is the effective intervalley deformation potential. ${ }^{19}$ To fit the FCA spectra at $2 \mathrm{ps}, D_{X_{6} X_{7}}$ is treated as an adjustable parameter. The results for the best fit are shown as the dashed line in Fig. 1. The best fit to the data is obtained for a value of $D_{X_{6} X_{7}}=3.2 \mathrm{eV} / \AA$. A mobility of $\mu=1.4 \mathrm{~cm}^{2} / \mathrm{V} \mathrm{s}$ was obtained for the $X_{7}$ valley electron population at the delay time of 2 ps. For the photoexcited high-energy, high-density electron-hole plasma generated in our experiment, the mobility of the $X$, valley electrons is significantly reduced due to strong, relatively unscreened ${ }^{18}$ intervalley scattering to the higher effectivemass $X_{6}$ valley. The intervalley scattering rate of $X_{6}$ valley electrons is not as great since few $X_{6}$ valley electrons are energetic enough to scatter into the $X_{7}, \Gamma_{6}$, or $L_{6}$ valleys.

For longer delay times the electrons have transferred to the lowest-energy $X_{6}$ valley. The dominant scattering mechanism giving rise to the FCA at these long delay times is electron-hole scattering of holes with electrons in the $X_{6}$ valley. From the magnitude of the FCA the mobility of the $X_{6}$ valley electrons is estimated to be $\mu=5.5$ $\mathrm{cm}^{2} / \mathrm{V}$ s at 30 ps.

The transient mobility of the electrons was then calculated by the above procedure for each delay time that the absorption at $\lambda_{\text {probe }}=4.7 \mu \mathrm{m}$ was measured. The result of this calculation is shown in Fig. 4. For the first data point, the electron mobility is $117 \mathrm{~cm}^{2} / \mathrm{V} \mathrm{s}$, which is close

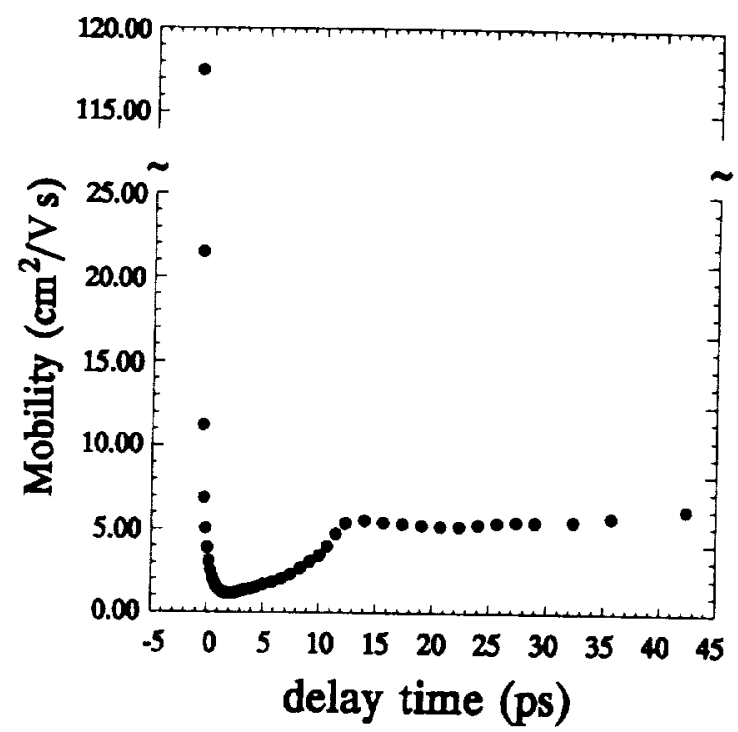

FIG. 4. Transient mobility of the electrons calculated as explained in the text.

to the steady-state value of $120 \mathrm{~cm}^{2} / \mathrm{V} \mathrm{s}$, as quoted in the literature. As the population of the $X_{7}$ valley builds up, the electron mobility rapidly drops to a value of 1.4 $\mathrm{cm}^{2} / \mathrm{Vs}$ due to $X_{7} \rightarrow X_{6}$ intervalley scattering. As the $X_{7}$ valley population decays and the $X_{7} \rightarrow X_{6}$ intervalley scattering slows down, the mobility recovers to a value of $5.5 \mathrm{~cm}^{2} / \mathrm{V} \mathrm{s}$, and from this time on the mobility is limited by electron-hole scattering due to the high excitation density. The electron mobility will only slowly increase to its steady-state value on the same time scale as electron-hole recombination or carrier diffusion.

In summary, measurements of the induced IR (2.5-5.5 $\mu \mathrm{m})$ absorption, due to photoexcited high-energy, highdensity electrons in the satellite and upper conductionband valleys in GaP, were performed. From a fit to the IR-absorption spectra insight into the dynamics of the relaxation of the hot electrons is obtained. It was determined that at early delay times, $\sim 2 \mathrm{ps}$, the dominant scattering mechanisms are intervalley scattering of electrons from the upper conduction-band, $X_{7}$ valley to the lower conduction-band, $X_{6}$ valley, and electron-hole scattering. The $X_{7}$ valley population is depleted within 5 ps, and for longer times the dominant scattering mechanism is electron-hole scattering of holes with $X_{6}$ valley electrons. From the magnitude of the induced FCA the $X_{7} \rightarrow X_{6}$ effective intervalley scattering deformation potential and the transient mobility of the hot electrons were estimated.

We would like to thank Brian Fitzpatrick of Optical Semiconductors for the GaP samples. This research is supported by NASA under Grant No. NAG3-1490 and the New York State Technology Foundation Grant. 
${ }^{1}$ A. F. Gibson, M. F. Kimmit, S. Kothari, C. B. Hatch, and A. A. Serafetinides, Appl. Phys. Lett. 30, 36 (1977).

${ }^{2}$ T. P. Pearsall, IEEE J. Quantum Electron. QE-16, 709 (1980).

${ }^{3}$ W. B. Wang (private communication).

${ }^{4}$ E. M. Conwell, in Solid State Physics, Advances in Research and Applications, edited by F. Seitz, D. Turnbull, and $\mathbf{H}$. Ehrenreich (Academic, New York, 1967), Suppl. 9.

${ }^{5}$ M. J. Kann, A. M. Kriman, and D. K. Ferry, Phys. Rev. B 41, 12659 (1990).

${ }^{6}$ W. B. Wang, K. Shum, R. R. Alfano, D. Szmyd, and A. J. Nozik, Phys. Rev. Lett. 68, 662 (1992).

${ }^{7}$ A. S. Jordan, J. Appl. Phys. 51, 2218 (1980).

${ }^{8}$ W. B. Wang, N. Ockman, M. A. Cavicchia, and R. R. Alfano, Appl. Phys. Lett. 57, 395 (1990).

${ }^{9}$ J. W. Hodby, Proc. Phys. Soc. 82, 324 (1963).

${ }^{10}$ J. Pankove, Optical Processes in Semiconductors (Dover, New York, 1971), Chap. 3.
${ }^{11}$ M. A. Cavicchia and R. R. Alfano, Proc. SPIE 2142, 128 (1994).

${ }^{12}$ C. Harbeke, O. Madelung, and U. Rössler, in Semiconductors, edited by $O$. Madelung, Landolt-Börnstein, New Series, Group III, Vol. 17, Pt. a (Springer-Verlag, New York, 1982).

${ }^{13}$ M. A. Cavicchia and R. R. Alfano, Phys. Rev. B 48, 5696 (1993).

${ }^{14}$ K. Seeger, Semiconductor Physics (Springer-Verlag, Berlin, 1982), Chap. 11.

${ }^{15}$ P. N. Saeta, J. F. Federici, B. I. Greene, and D. R. Dykaar, Appl. Phys. Lett. 60, 1477 (1992).

${ }^{16}$ M. A. Cavicchia and R. R. Alfano (unpublished).

${ }^{17}$ T. P. Mclean and E. G. S. Paige, J. Phys. Chem. Solids 16, 220 (1960).

${ }^{18}$ E. J. Yoffa, Phys. Rev. B 23, 1909 (1981).

${ }^{19}$ S. Zollner, S. Gopalan, and M. Cardona, J. Appl. Phys. 68, $1682(1990)$. 
